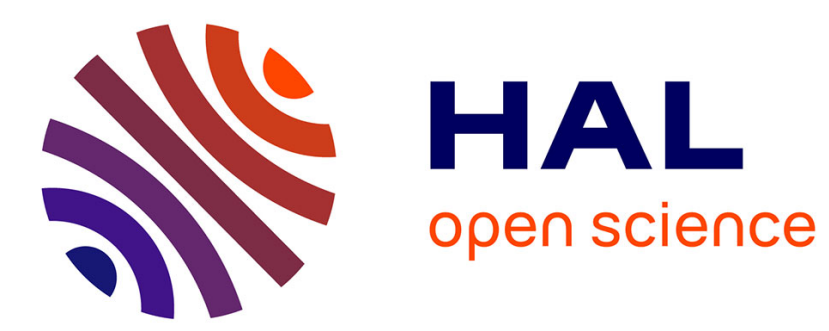

\title{
Les batteries au lithium à électrolyte polymère. Les activités de R\&D à Hydro-Québec
}

\author{
B. Kapfer
}

\section{To cite this version:}

B. Kapfer. Les batteries au lithium à électrolyte polymère. Les activités de R\&D à Hydro-Québec. Journal de Physique IV Proceedings, 1994, 04 (C1), pp.C1-245-C1-251. 10.1051/jp4:1994118 jpa00252460

\section{HAL Id: jpa-00252460 https://hal.science/jpa-00252460}

Submitted on 1 Jan 1994

HAL is a multi-disciplinary open access archive for the deposit and dissemination of scientific research documents, whether they are published or not. The documents may come from teaching and research institutions in France or abroad, or from public or private research centers.
L'archive ouverte pluridisciplinaire HAL, est destinée au dépôt et à la diffusion de documents scientifiques de niveau recherche, publiés ou non, émanant des établissements d'enseignement et de recherche français ou étrangers, des laboratoires publics ou privés. 


\title{
Les batteries au lithium à électrolyte polymère. Les activités de $R \& D$ à Hydro-Québec
}

\section{B. KAPFER}

Institut de Recherche d'Hydro-Québec, 1800 Montee Sainte-Julie, Varennes, Québec J3X 1S1, Canada

\begin{abstract}
Hydro-Quebec, in close collaboration with the French CNRS and the INP de Grenoble for the LIES laboratory, has been involved in electrochemical research and development activities on a rechargeable lithium polymer battery since 1979. Major improvements in the battery performance in terms of cycling and energy density led to a joint-venture with Yuasa, a Japanese battery manufacturer, in 1990. Room-temperature activities, dedicated to electronics, are carried out by the Japanese and electric vehicle and stationary applications by the Canadians. Hydro-Quebec's R\&D activities are presently focused on the $2-\mathrm{kWh}$ battery program. The main goal is to prove the feasibility of this technology, assess the unit operation required for production by an integrated pilot plant, and demonstrate the technology on the 2-kWh scale. The IREQ laboratory in Varennes is responsible for film preparation and characterizations (cycling, post-mortem, thermal management...) and LTEE laboratory in Shawinigan for cell assembly and packaging.
\end{abstract}

\section{Généralités}

Hydro-Québec, en étroite collaboration avec le CNRS et l'INP de Grenoble au nom du LIES, est impliqué depuis 1979 dans des activités de recherche et développement pour des batteries au lithium à électrolyte polymère. Cette technologie, dont le concept a été proposé par M.B. Armand, repose sur l'idée originale de l'utilisation d'un polymère conducteur par les ions lithium comme électrolyte au sein d'un accumulateur au lithium' ${ }^{1}$. Ce polymère, en l'occurrence un polyéther appelé polyoxyde d'éthylène, est stable à l'oxydation du lithium et il solubilise des sels de lithium, s'assurant ainsi une certaine conduction ionique nécessaire à sa fonction d'électrolyte. Comme le montre la figure 1, la faible conductivité observée est largement compensée par deux des aspects fondamentaux de la batterie à savoir, d'une part l'épaisseur de l'électrolyte très largement inférieure à $100 \mu \mathrm{m}$ et les grandes surfaces mises en jeu, et d'autre part la température d'opération de l'ordre de $100^{\circ} \mathrm{C}$ qui permet d'obtenir les valeurs requises tant pour la conductivité de l'électrolyte que pour les coefficients de diffusion dans les électrodes. Cette batterie tout solide, se singularise essentiellement par sa grande densité d'énergie, caractéristique de l'usage des métaux alcalins, par l'absence d'autodécharge et par une très grande cyclabilité. Elle a été perçue dès le début, dans le cadre de la diversification des usages de l'électricité, comme une application très prometteuse pour le développement de batteries de grande dimension aussi 
bien pour le véhicule électrique que pour des installations stationnaires, comme par exemple le nivellement de charge, les sources de courant ininterrompues et la téléphonie.

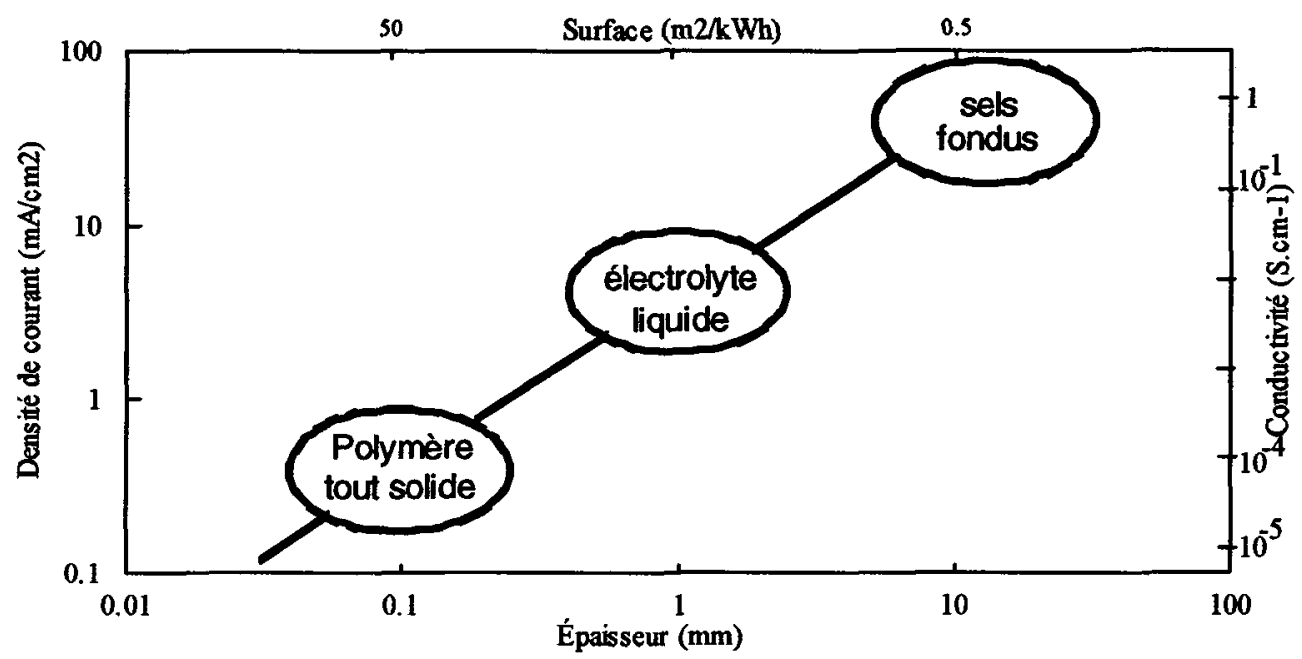

Figure 1: comparaison de différentes technologies (100 W/, $\eta=95 \%)$

\section{Des progrès majeurs}

Les premiers travaux ont essentiellement porté sur le développement de nouveaux matériaux, polymères, sels et additifs (appelés plastifiants), permettant d'améliorer la conductivité de l'électrolyte et par conséquence de permettre l'utilisation de tels accumulateurs à des températures de plus en plus basses. D'un point de vue plus fondamental, cela signifiait le développement de complexes sel-polymère présentant une température de transition vitreuse $(\mathrm{Tg})$ la plus basse possible, conduisant ainsi à une vaste zone d'amorphicité dans laquelle l'évolution des propriétés de conduction en fonction de la température est la moins marquée ${ }^{2}$. Il en a résulté l'apparition de copolymères dont la composition, basée sur celle du polyoxyde d'éthylène, a été modifiée par l'introduction le long des chaînes de défauts tels que des groupements différents ou des chaînes latérales ${ }^{3-4}$. Le développement de nouveaux sels de lithium ayant une meilleure solubilité et présentant un large domaine de stabilité électrochimique a constitué un autre axe majeur de recherche. Il faut en particulier signaler la mise au point d'un sel, le trifluoro-sulfonyl-imidure de lithium (LiTFSI), produit maintenant par la compagnie américaine 3M sous licence Hydro-Québec, qui est aujourd'hui largement étudié pour l'ensemble des technologies de batteries au lithium. La plupart des plastifiants traditionnels basés sur le carbonate de propylène (PC), bien que conduisant à des augmentations très importantes de la conductivité, ont des problèmes de stabilité avec les autres composantes et ne présentent donc que peu d'intérêt dans un accumulateur ${ }^{5}$. Hydro-Québec a néanmoins développé une famille de plastifiants électrochimiquement compatibles avec l'ensemble des composantes de l'accumulateur et dont le gain en conductivité, bien qu'inférieur à celui du PC par exemple, reste très important. Comme le montre la figure 2 , il convient toutefois de noter qu'au dessus de $60^{\circ} \mathrm{C}$, la différence entre un polymère sec et plastifié est peu significative. Cette température est présentement la température d'opération minimale nécessaire pour satisfaire les spécifications de 
puissance requises pour des applications de type véhicule électrique. Ces considérations sont à la base des choix technologiques qui ont orienté le programme de $R \& D$ présentement en cours qui privilégie l'utilisation de polymères secs pour les applications de type traction opérant autour de $60^{\circ} \mathrm{C}$ et celle de polymères plastifiés pour celles opérant aux plus basses températures (électronique et informatique).

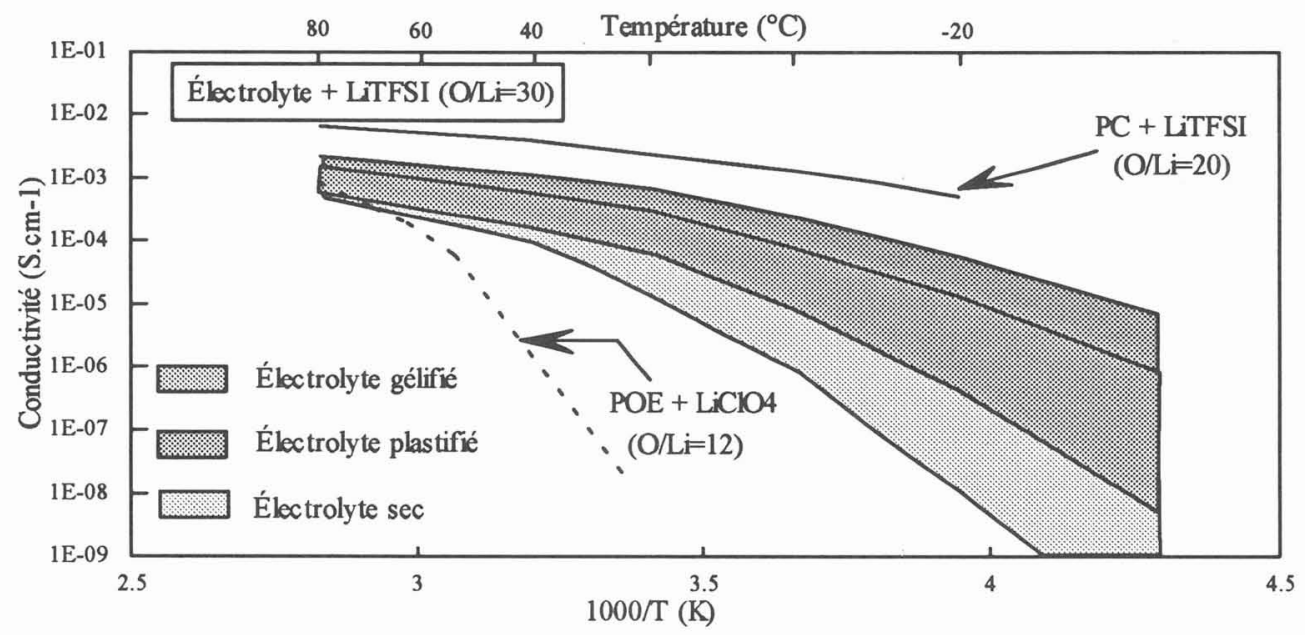

Figure 2: Influence de plastitiants sur la conductivite

La figure 3 décrit les progrès qui ont été faits dans le cadre de l'étude sur la traction électrique. La température d'opération, initialement de l'ordre de $100-150^{\circ} \mathrm{C}$, a décrû régulièrement pour se stabiliser à environ $60^{\circ} \mathrm{C}$, le contenu énergétique et le nombre cycles réalisés ont atteint respectivement $150 \mathrm{Wh} / \mathrm{kg}$ et 1000 cycles. La protection légale de ces travaux est par ailleurs concrétisée par près de 50 brevets couvrant tant les aspects fondamentaux que les procédés. En 1990, la signature d'une entente avec la compagnie japonaise Yuasa voyait les aspects "applications grande consommation", opérant autour de la température ambiante et couvrant principalement l'électronique et l'informatique, assurés par la partie japonaise et ceux visant la traction et le stationnaire par la partie québécoise.

\section{Situation actuelle}

Depuis 1991, les activités de R\&D peuvent être regroupées selon trois axes principaux qui traduisent les diverses facettes des travaux qui vont des considérations les plus fondamentales à celles beaucoup plus appliquées qui caractérisent toute phase de démonstration.

\section{Les projets de recherche (Matériaux et Défense)}

La collaboration avec diverses Universités (Grenoble en France, Purdue University aux États-Unis, Montréal et Sherbrooke au Canada) constitue une grande partie des travaux de recherche qui s'effectue dans le cadre de ce projet. Elle se fait sous la forme aussi bien de contrats de recherche que de financement de travaux de maîtrise ou de thèse. La compréhension des mécanismes fondamentaux régissant cette technologie, l'évaluation de nouveaux concepts et le développement de nouveaux polymères et sels représentent un apport essentiel aux progrès de cette filière. Hydro-Québec pour sa 
part assure l'évaluation dans des conditions standards de tous les nouveaux matériaux issus de ces collaborations. Un effort plus particulier est par ailleurs fait pour comprendre les mécanismes de vieillissement des accumulateurs, vieillissement qui se traduit essentiellement par une dégradation sensible des caractéristiques de puissance.

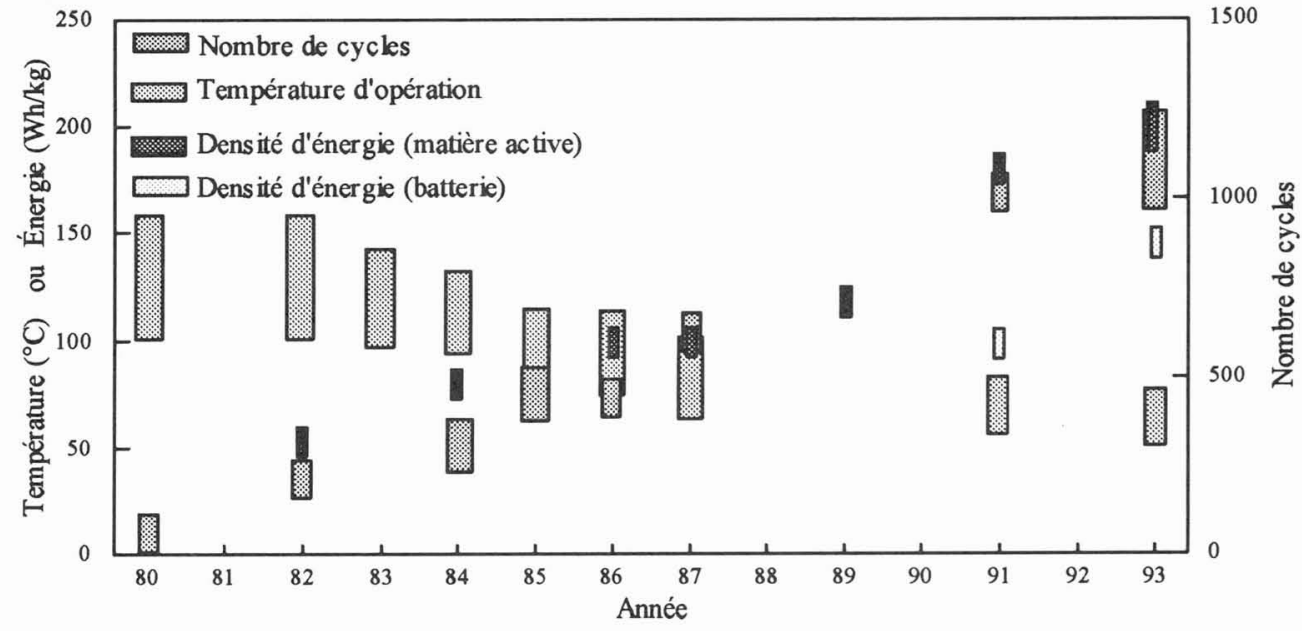

Figure 3: progrès des performances ACEP dans le temps

\section{Le projet "ACEP traction"}

Cette activité fait le lien entre la recherche et l'ensemble des activités de développement présentement en cours. Elle fait les recommandations pour les nouveux composés et les nouvelles formulations et permet leur intégration dans les procédés au niveau du développement. Ses principaux objectifs sont:

- amélioration des performances au niveau de la cellule de laboratoire dans la gamme $40-80^{\circ} \mathrm{C}$ en terme de contenu énergétique, de puissance soutenue et instantanée selon les spécifications propres au véhicule électrique et enfin de vie utile,

- amélioration des compositions et des composés utilisés dans le programme " $2 \mathrm{kWh}$ " en terme d'excès de lithium, d'épaisseur d'électrolyte, de composition d'électrode et d'optimisation des collecteurs de courant,

- intégration de nouveaux produits tels que cathodes à hauts voltage ou évaluation d'alternatives aux anodes actuelles,

- importante activité de caractérisations en cyclage (séries, profils, ...),

- analyses par spectroscopie d'impédance et analyses post-mortem (MEB, EDAX, SIMS, Auger, $\ldots)$,

- évaluation comparative de la technologie ACEP en terme de consommation électrique, autonomie, durée de vie et recyclabilité pour ne citer que les principaux.

\section{Le programme "ACEP 2 kWh"}

Ce programme constitue la partie la plus importante des activités en cours. Il vise principalement à prouver la faisabilité de cette technologie en terme de procédés, performances et sécurité, à évaluer sous 
forme de bancs d'essais chacune des étapes nécessaires dans un procédé continu d'élaboration d'une batterie, et enfin à démontrer la filière "batterie au lithium à électrolyte polymère" à l'échelle d'une batterie de $2 \mathrm{kWh}$, énergie qui correspond à des marchés spécifiques (fauteuils pour handicapé, source de courant ininterruptible par exemple) et qui représente une étape critique avant la batterie pour la voiture électrique.

Les unités élémentaires qui composeront ce prototype seront cylindriques, de la taille d'une cannette de bière et auront un contenu énergétique est de l'ordre de $100 \mathrm{Wh}^{6}$. La configuration retenue qui privilégie l'aspect "parallèle" à celui "série" au sein de ces unités présente divers avantages. Elle permet dans la mesure où une seule longueur de film est enroulée autour d'un mandrin, d'abord une élaboration rapide et facile par des procédés automatiques, ensuite une gestion thermique plus favorable par la création de raccourcis électriques et thermiques à chacune des extrémités et d'éviter le problème du balancement des unités en série qui est encore considéré comme un aspect recherche, et enfin d'atteindre les objectifs de densité d'énergie acceptables pour les applications traction. Deux autres formats, l'enroulement plat et l'empilement zig-zag (figure 4), sont présentement en cours de développement. Ils représentent des alternatives technologiques qui permettront essentiellement une augmentation du contenu volumique à la fois des unités et de la batterie par la réduction des espaces vides.

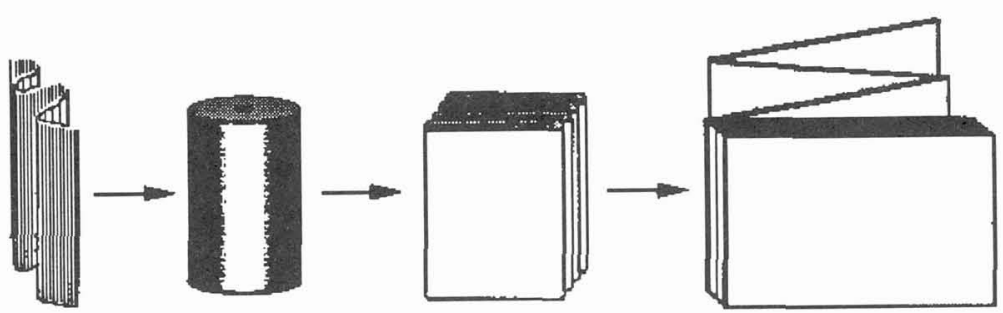

Figure 4: unités élémentaires développées dans le cadre du programme "ACEP 2 kWh"

Cette activité est répartie entre le site de l'IREQ proprement dit où se fait l'élaboration des composantes et les diverses caractérisations, et le laboratoire des technologies électrochimiques et des électrotechnologies (LTEE) à Shawinigan pour l'assemblage et l'emballage.

Dans la phase actuelle, la production essentielle consiste en des unités de l'ordre de $10 \mathrm{Wh}$. Cette dimension répond de manière appropriée aux besoins d'optimisation requis aussi bien pour la mise au point des procédés d'élaboration ou d'emballage que pour les choix de design ou de matériaux et pour les diverses études de caractérisations.

Les objectifs en terme de contenu énergétique sont décrits dans le tableau 1. Les valeurs fournies incluent non seulement la matière active, mais aussi l'ensemble des pertes résultant de l'emballage, des collecteurs de courants et de la gestion thermique.

La progression du contenu au niveau des cellules élémentaires correspond à une optimisation à la fois technologique et électrochimique. Pour les besoins de ce programme, un total de 150 de ces unités devront être produites. Un certain nombre de cellules de la génération "1993" seront prélevées pour la réalisation des premiers prototypes de $2 \mathrm{kWh}$, celles de la génération "1994" pour ceux de la phase ultérieure. Il convient enfin de noter qu'en terme de longueur de films, la production du premier prototype aura nécessité au-delà de $12 \mathrm{~km}$ de chacun des films composant la batterie. 
Tableau 1: Objectifs en terme de contenu énergétique pour le programme "ACEP $2 \mathrm{kWh}$ "

\begin{tabular}{|c|c|c|c|c|}
\hline & \multicolumn{3}{|c|}{ Programme $2 \mathrm{kWh}$} & \multirow{2}{*}{\begin{tabular}{|c|} 
Phase ultérieure \\
1995-1998
\end{tabular}} \\
\hline & 1991-1992 & 1993 & 1994 & \\
\hline $\begin{array}{c}\text { Cellules } \\
\text { élémentaires } \\
\text { (150 unités) }\end{array}$ & $\begin{array}{c}100 \mathrm{Wh} \\
133 \mathrm{Wh} / \mathrm{l} \\
104 \mathrm{Wh} / \mathrm{kg}\end{array}$ & $\begin{array}{l}144 \mathrm{Wh}: \\
192 \mathrm{Wh}: \\
137 \mathrm{Wh} / \mathrm{kg} \text {. }\end{array}$ & 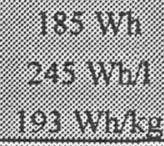 & \\
\hline $\begin{array}{c}\text { Batteries } 2 \text { kWh } \\
\text { (2 à } 3 \text { unités) }\end{array}$ & & & 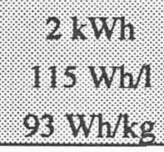 & \\
\hline $\begin{array}{c}\text { Batteries } 40 \mathrm{kWh} \\
\text { ou } \\
\text { Batteries } 2 \mathrm{kWh}\end{array}$ & & & & 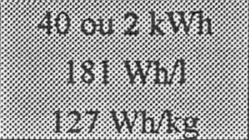 \\
\hline
\end{tabular}

La figure 5 permet une comparaison entre les performances des diverses technologies. Elle fait clairement ressortir l'avantage des batteries ACEP en terme de contenu énergétique.

La sécurité, associée la gestion thermique, représente un aspect crucial de cette technologie. Des études par calorimétrie (DSC) ont montré une différence fondamentale de comportement entre les électrolytes liquides et polymériques? ${ }^{7}$. En effet, à la température de fusion du lithium de l'ordre de $180^{\circ} \mathrm{C}$, aucun pic exothermique n'apparaît pour les électrolytes polymériques, démontrant une certaine stabilité en milieu fortement réducteur typique du lithium fondu. Ce comportement a par la suite été vérifié sur une unité de $10 \mathrm{Wh}$ où, dans le cas d'un court-circuit interne franc, la température monte d'abord rapidement aux alentours de $180-190^{\circ} \mathrm{C}$, s'y stabilise quelques minutes pour décroître enfin lentement. Par ailleurs, d'importants travaux de simulation ont été entrepris afin de connaître de manière approfondie le comportement thermique de cette technologie, travaux dont les applications concernent autant l'aspect sécurité que celui de la gestion thermique proprement dite d'abord dans une unité élementaire, ensuite dans le prototype.

\section{Perspectives et conclusions}

Le programme ACEP $2 \mathrm{kWh}$ devra démontrer de manière décisive la faisabilité technologique et permettre la mise en place à court terme d'une usine pilote. La production des premières unités de 100 Wh en 1992 et la production actuelle de nombreuses unités de 10 Wh pour les travaux de validation sont autant de facteurs qui permettent à Hydro-Québec d'envisager l'avenir de cette activité avec un certain optimisme.

Ce programme est largement soutenu par une forte activité de recherche où l'accent est mis sur la compréhension des mécanismes de vieillissement qui caractérisent actuellement cette technologie, sur l'étude de nouveaux matériaux d'électrodes et enfin sur le développement d'électrolytes à conductivité améliorée. 


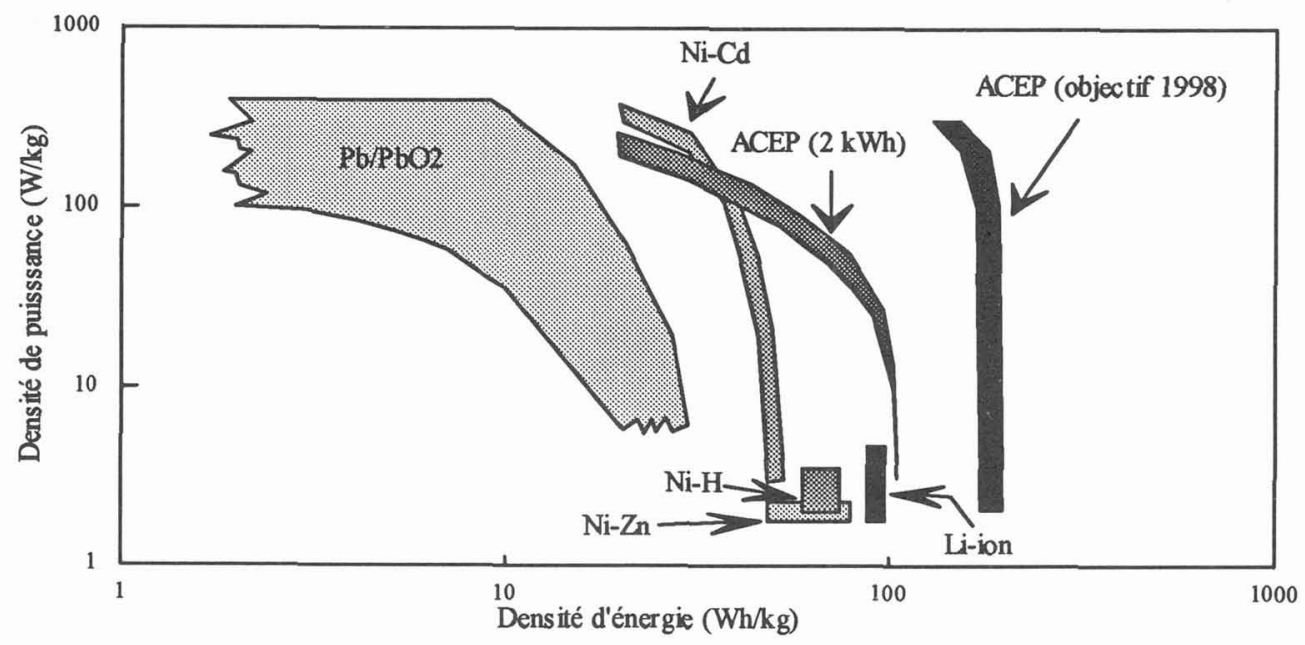

Figure 5: comparaison des diverses technologies d'accumulateurs.

L'importance de cette technologie est maintenant largement reconnue à travers le monde où des programmes très importants sont financés au niveau européen (ECC-Joule2), japonais (MITI) et nordaméricain (USABC). Ce dernier programme, financé par les 3 grands manufacturiers de l'automobile (Ford, Chrysler et GM), le US Department of Energy et I'EPRI, a déjà attribué une subvention au groupe Grace. Hydro-Québec, associé à un manufacturier américain, est en négociation avec ce consortium depuis 1991. Enfin, le groupe Valence, qui a acheté les activités de MEAD et ERLInnovision, fonctionne sur financement privé.

1 M.B. Armand, J.M. Chabagno et M.J. Duclot, in Fast Ion Transport in Solids, édité par P. Vashista, J. N. Mundy et G.K. Shenoy, p. 131, Elsevier, New York, 1979

2 M. Gauthier, M.B. Armand et D. Muller, in Electroresponsive Molecular and Polymeric Systems, édité par T.A. Skotheim, p. 40, Marcel Dekker, Inc., new York et Bale, 1988

3 P.E. Harvey, 4th International Meeting on Lithium Batteries, Vancouver, Canada, Mai 1986

4 H.R. Allcock, P.E. Austin, T.X. Neenan, J.T. Sisko, P.M. Blonsky et D.F. Shriver, Macromolecules, 19, p. 1508, 1986

5 B. Kapfer, Workshop on Rechargeable Lithium Polymer Batteries, Proceedings Report RP241522, Kirkland, Washington, Novembre 1990

6 M. Gauthier et al., 10th International Seminar on Primary and Secondary Battery Technology and Application,Deerfield Beach, Florida, USA, Mars 1993

7 Y. Choquette et al., 6th International Meeting on Lithium Batteries, Münster, Allemagne, Mai 1992 